\title{
Wie wirken Standards und Normen im Recht?
}

Standards sind so etwas wie logische Bausteine der Informationsgesellschaft. Sie sind zum einen Voraussetzung dafür, dass viele unterschiedliche IT-Systeme reibungslos zusammenarbeiten. Zum anderen entsteht durch Standardisierung auch erheblicher Nutzen in den verschiedensten Bereichen der Gesellschaft: Unternehmen etwa profitieren durch die Möglichkeit schnellerer Produktentwicklung und Markteinführung, Innovationspotential wird beschleunigt, internationaler Handel wird vereinfacht und transparenter, die rechtliche Regelung komplexer technischer Tatbestände unterstützt und nicht zuletzt bewirken Standards auch mehr Sicherheit und Risikominimierung für alle Marktteilnehmer.

Gerade im Kontext der IT-Sicherheit kommt Standards wachsende Bedeutung zu. Nicht nur, um Protokolle, Verschlüsselungs- und/oder Authentifizierungsverfahren im Sinne optimierter Interoperabilität zu harmonisieren. Vielmehr bieten verschiedenste Standards Unternehmen und Behörden interessante Möglichkeiten, mit erprobten Methoden ressourcenschonende Prozesse für ein Risiko- und IT-Sicherheitsmanagement zu implementieren. Im vom BITKOM und DIN gemeinsam herausgegebenen Kompass der IT-Sicherheitsstandards ${ }^{1}$ sind die für den IT-Sicherheitsbereich einschlägigen Standards umfassend dokumentiert.

Die in der Praxis bedeutendste und am häufigsten anzutreffende Ausprägung von Standards sind Normen. Der Begriff der Norm ist selbst durch eine Norm definiert, der DIN EN 45020: Ein „Dokument, das mit Konsens erstellt und von einer anerkannten Institution angenommen wurde und das für die allgemeine und wiederkehrende Anwendung Regeln, Leitlinien oder Merkmale für Tätigkeiten oder deren Ergebnisse festlegt (...).“

1 Der aktuelle Kompass der IT-Sicherheitsstandards liegt in 4. Aufl. vom August 2009 vor (kostenloser Download auf der Website des BITKOM www. bitkom.org oder des DIN www.din.de )
Die Begriffe Standards und Normen werden in der Praxis nicht immer einheitlich gebraucht. Man kann grob folgende Unterscheidung zugrunde legen: Standards sind in einem Konsensprozess entstandene Spezifikationen, wobei hinsichtlich des Konsensrahmens beträchtliche Nuancen möglich sind. Normen sind solche Standards, bei denen der Konsens in einem geregelten Beteiligungsverfahren hergestellt wurde. Ein solches Verfahren erfolgt auf nationaler Ebene, also in Deutschland gemäß den Regelungen von DIN 820-4. Dabei haben insbesondere die interessierten Fachkreise Gelegenheit zur aktiven Mitwirkung an der Norm und können so ihre Expertise und Interessen im Sinne einer optimierten Konsensfindung und späterer Akzeptanz der Norm gezielt einbringen ${ }^{2}$. Interessant in diesem Zusammenhang ist die Praxis des Bundesamtes für Sicherheit in der Informationstechnik (BSI), technische Regeln in einem internen Prozess, ohne ein für die Standardsetzung typisches Konsensverfahren, als sog. „Technische Richtlinien" zu erstellen. Auch wenn diese Praxis nun in $\$ 8$ Abs. 2 des BSI-Gesetzes eine Rechtsgrundlage erhalten hat, lassen sich die Technischen Richtlinien des BSI nicht in die klassische Typik der Standards und Normen einordnen.

Die große Bedeutung von Normen im Wirtschaftsverkehr und im Rechtsleben erklärt sich zunächst aus der der deutlichen Vereinfachung, die ein Bezug auf Normen für die Konkretisierung von vertraglich vereinbarten Leistungen oder Merkmalen von Ausschreibungsinhalten haben kann.

2 In diesem Sinne jüngst auch Neelie Kroes, Vizepräsidentin der EU Kommission und verantwortlich für die Digital Agenda der EU in einer Rede am 10. Juni 2010 (SPEECH/10/300): ,... In the case of a formal standard the specification is drawn up by a standardsetting organisation pursuant to a welldefined process. For example, the process should be open and transparent and allow for participation of all interes ted stakeholders, not the least to ensure buy-in and market acceptance for the resulting standards...."
Die eigentliche Wirkung von Normen im Recht entfaltet sich aber über sog. Technikklauseln. Denn nach ständiger Rechtsprechung sind „...DIN-Normen [sind] keine Rechtsnormen, sondern private technische Regelungen mit Empfehlungscharakter. Sie können die anerkannten Regeln der Technik wiedergeben oder hinter diesen zurückbleiben. ${ }^{\text {"S Somit }}$ kommt dem Begriff der ,allgemein anerkannten Regeln der Technik“ eine zentrale Bedeutung zu. Dies sind Regeln, die wissenschaftlich als theoretisch feststehend und richtig anerkannt sind, in der Praxis bei nach aktuellem Kenntnisstand ausgebildetem Personal üblicherweise bekannt sind und sich aufgrund längerer praktischer Erfahrung bewährt haben. Die Einhaltung von DIN-Normen löst nur eine widerlegbare Vermutung aus, dass damit die allgemein anerkannten Regeln der Technik eingehalten sind. Diese Sichtweise trägt vor allem dem Umstand Rechnung, dass Normen nicht immer aktuell sind und nicht sein können und sich auch nicht immer in der Praxis langfristig bewährt haben.

Für die Rechtssetzungspraxis immer wieder relevant ist, ob Rechtsnormen (Gesetzen oder Verordnungen) "gleitend“ auf Normen verweisen dürfen, typischer Weise durch den Zusatz ,in der jeweils geltenden Fassung“. Bei derartigen Verweisen kann der Normgeber die künftige Entwicklung der Bezugsnorm nicht selbst bestimmen. Der verfassungsrechtlich verankerte Gesetzesvorbehalt verlangt aber, dass die wesentlichen Grundzüge vom Normgeber selbst zu regeln sind. Deshalb sind gleitende Verweise auf private Regelwerke (z. B. DIN-Normen) aus verfassungsrechtlichen Gründen nicht zulässig. Normgeber dürfen ihre Rechtsetzung auch nicht indirekt auf Private übertragen ${ }^{4}$.

3 BGH - Az.: VII ZR 184/97, Urteil vom 14.05.1998 (BGHZ 139, 16)

4 Dazu Handbuch der Rechtsförmlichkeit, Herausgegeben vom Bundesministerium der Justiz, 3., neu bearbeitete Auflage 2008, dort RZ 246 ff. URL: http://hdr.bmj.de/ 